\title{
Cortex-involved Ischemic Stroke and Subtypes of Atrial Fibrillation
}

\author{
Shuya Li, Xiaomeng Yang, Xingquan Zhao, Liping Liu, Yong Jiang, Zixiao Li, Yilong \\ Wang, Yongjun Wang
}

BACKGROUND: Cortex-involved (Both cortical and corticalsubcortical) lesions are typically caused by embolic stroke, of which Atrial fibrillation(AF) is the common cause.

OBJECTIVE: The aim of this study was to investigate the associations between cortex-involved stroke, vascular risk factors and the subtypes (discovery time and duration) of AF. DESIGN, SETTING, AND PARTICIPANTS: This is a prespecified imaging study of the China Atrial Fibrillation Screening in Acute Ischemic Stroke Patients (CRIST) trial. Between October 2013 and June 2015, 1511 acute ischemic stroke or transient ischemic attack (TIA) patients within 7 days after stroke onset were enrolled in this prospective, multicenter cohort, Cross-sectional study at 20 Chinese hospitals. The primary aim of CRIST trial is to define the true frequency of AFassociated stroke. The final analysis of this substudy included 243 AF patients with required Magnetic Resonance Imaging (MRI) sequences. AF were diagnosed by 6-day Holter monitoring and electrocardiograph(ECG) and classified by duration of 24 hours.

METHODS: Two stroke specialists blinded to the clinical information reviewed MRI(Diffusion-weighted MR imaging, FLuid Attenuated Inversion Recovery FLAIR, T1 weighted imageT1WI, T2 weighted imageT2WI). The third stroke specialists, also blinded to the clinical information, assessed the conflicts. Adjusted large artery atherosclerosis as confounding factor, the associations between Cortex-involved lesions, vascular risk factors and the subtype of AF were evaluated by univariate and multivariate regression analysis.
RESULTS: Of 243 acute ischemic stroke patients with AF, 190 were known AF and 53 were new-detected AF. there were 28 with AF persistent more than 24 hours and 25 persistent less than 24hours in newly detected AF. Cortex-involved lesions were significantly related to newly detected AF persistent more than 24 hours $(\mathrm{OR}=4.517,95 \% \mathrm{Cl} 1.490-13.696, \quad \mathrm{P}=0.0077)$, Proteinuria (OR=3.431, 95\% $\mathrm{Cl} 1.530-7.692, \quad \mathrm{P}=0.0213)$ and glycosylated hemoglobin(OR=0.632, $95 \% \mathrm{Cl} \quad 0.464-0.861$, $\mathrm{P}=0.0036)$.

CONCLUSIONS: Compared to previously known AF, Newly detected AF persistent more than 24 hours was robustly associated with cortex-involved ischemic stroke.

KEY WORDS: Atrial fibrillation, Ischemic stroke, Prolonged ECG monitoring, MRI

Trial registration: NCT02156765

Table. Risk factors for Cortex-involved infarct

\begin{tabular}{|l|l|l|}
\hline Risk factors, $\mathrm{n}(\%)$ & OR & $95 \% \mathrm{CI}$ \\
\hline Proteinuria & 3.431 & $1.530-7.692$ \\
\hline GHB & 0.734 & $0.610-0.883$ \\
\hline New diagnosed AF & 4.435 & $1.888-10.415$ \\
\hline \multicolumn{1}{|c|}{$\mathrm{AF}>24 \mathrm{~h}$} & 4.517 & $1.490-13.696$ \\
\hline
\end{tabular}

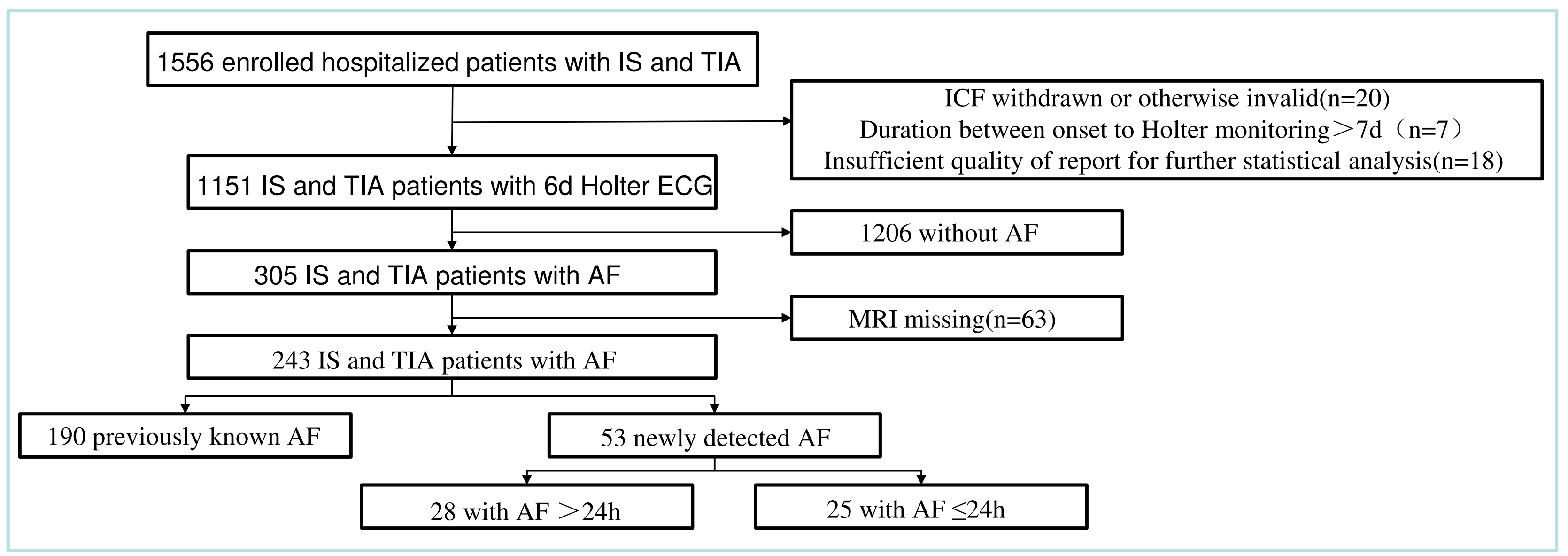

